\title{
A model updating method for truss structure using stepwise uniform design schemes considered primary factors
}

\section{Abstract}

Based on the feasibility and reliability of a forward analytical model updating method with uniform design having been proven, this paper studies the effectiveness of the method in the condition that structural measured information is uncertain and incomplete. By taking the experimental data of a steel truss as an example, this paper studies the method of experimental data processing, the determination of structural model with unknown parameters, the interval estimation of identification results and stepwise uniform design considered the correlation among identification parameters. The results show that the errors between the numerical results computed by the updated model and the experimental data are acceptable, which means the method in this paper is feasible and reliable. In the end, based on our experiences and lessons, we summarize a model updating method for complex structures using stepwise uniform design schemes considered the primary and secondary factors, and expound its computational steps.

\section{Keywords}

Truss Structure, Model Updating, Uniform Design, Factor.

\author{
Shilei Zhang ${ }^{\mathrm{a}, *}$ \\ Shaofeng Chen ${ }^{\text {b }}$ \\ Huanding Wang \\ Wei Wang \\ Zaixian Chen ${ }^{\mathrm{a}}$ \\ ${ }^{a}$ School of Civil Engineering, Harbin Institute \\ of Technology, Harbin 150090, China \\ ${ }^{\mathrm{b}}$ School of Transportation Science and \\ Engineering, Harbin Institute of Technology, \\ Harbin 150090, China \\ Received in 14 Oct 2012 \\ In revised form 08 Feb 2013 \\ Author email: zhanglei202202@163.com
}

\section{INTRODUCTION}

In order to guarantee the efficient operation of main communication arteries, it is very necessary to carry out model updating of large bridges built in these arteries so that assesses their safety performances. Model updating is a process that corrects structural original mechanical model by structural measured information. There are two basic thoughts for solving the problem of model updating: inverse identification and forward analysis. In case that structural excitation and responses are known, inverse identification is a process that identifies structural physical and geometric parameters or the matrix elements of structural dynamical equation. Inverse identification includes many algorithms such as the optimal matrix direct solution and the iteration method based on frequency- 
response function and so on, while above algorithms can be boiled down to solve the constrained optimization problem of inverse identification equation. The advantage of inverse identification is that its concept is clear, but the incompleteness of structural measured information and the impact of dynamic condensation result in that above constrained optimization problems are hard to be solved.

Forward analytical model updating (FAMU) is a process that establishes a database which contains structural real model and its corresponding responses based on researcher's mechanical knowledge, and finds structural real model from the database by the way of matching structural measured responses with each model responses in the database(Chang et al. (2002) and Khanmirza et al. (2011)). If the database is complete, the database will contain all possible states of the structure, which means that the database must contain structural real model. At this time, structural real model can be found from the database by matching structural responses. The advantage of FAMU is that it does not have to solve the constrained optimization problem, while the difficulty of inverse identification can be avoided. There have been some studies using FAMU together with neural networks (NN). Cheng (2010) derived a limit state function and defined a failure probability to assess the model of a suspension bridge by improving NN and genetic algorithm. Guajardo et al. (2010) studied how to update structural models when some new measured data were obtained. Basağa et al. (2011) identified the design parameters of a column by NN to make the frequencies of the column in accidence with the measured ones. Bakir et al. (2008) utilized NN and a global optimization technology to update the model of a two dimensional frame. Davoodi et al. (2012) updated a ball joint system by NN to analyze its nonlinear mechanical performances. Lu and Tu (2004) studied a two-step model updating method and corrected model parameters by frequency. Above mentioned studies prove the feasibility of FAMU, but FAMU also has its shortcoming.

If structural model is conditioned by $m$ factors and each factor has $n$ levels, a complete database for FAMU will contain $m^{n}$ models (Atalla and Inman (1998) and Davoodi et al. (2012)). At this time, in order to make a preparation for finding structural real model by matching responses, FAMU has to carry out $m^{n}$ times of computations to obtain structural responses under various states. When there are many factors and levels, the workload of FAMU will be very heavy (Ataei et al. (2005), Saaty and Vargas (1979), Unger and Könke (2011) and Zadeh (1965)). In order to reduce the workload of computation, uniform design (UD) is introduced into FAMU. We establish a FAMU method using UD, and the procedure of the method is shown as follow.

1) The principal factors and their levels of model updating are determined according to the actual structure which will be corrected.

2) A model updating plan is made by UD, and then $\mathrm{NN}$ will be trained by the plan.

3) Structural measured responses are input into the trained NN, while structural finite element model will be updated by the model parameters which are identified by the generalization ability of the NN.

The completeness and effectiveness of the FAMU method based on UD had been proven by the way of numerical simulation. The impact of multiple indices for model updating, such as vibration mode, frequency and displacement and so on, had been evaluated by a fuzzy analytic hierarchy process (Zhang et al. (2011)). The purpose of this paper is to study the feasibility of the FAMU 
method based on UD under the conditions that structural measured information is uncertain and incomplete.

\section{STATIC LOAD TEST DATA PROCESSING}

In order to check the FAMU method based on UD, the static load test of a steel truss is designed and its model parameters are updated. The size of the steel truss is $3.0 \mathrm{~m} \times 0.8 \mathrm{~m} \times 0.5 \mathrm{~m}$. The truss is installed on a compression-testing machine with $500 \mathrm{kN}$ shown in Figure 1. Five dial indicators whose accuracy is $200 \mu \varepsilon / \mathrm{mm}$ are used for measuring the deflections of the bottom chord. The sequence of the deflection measuring points is B1 $\sim \mathrm{B} 5$. The measured data are omitted to save space.

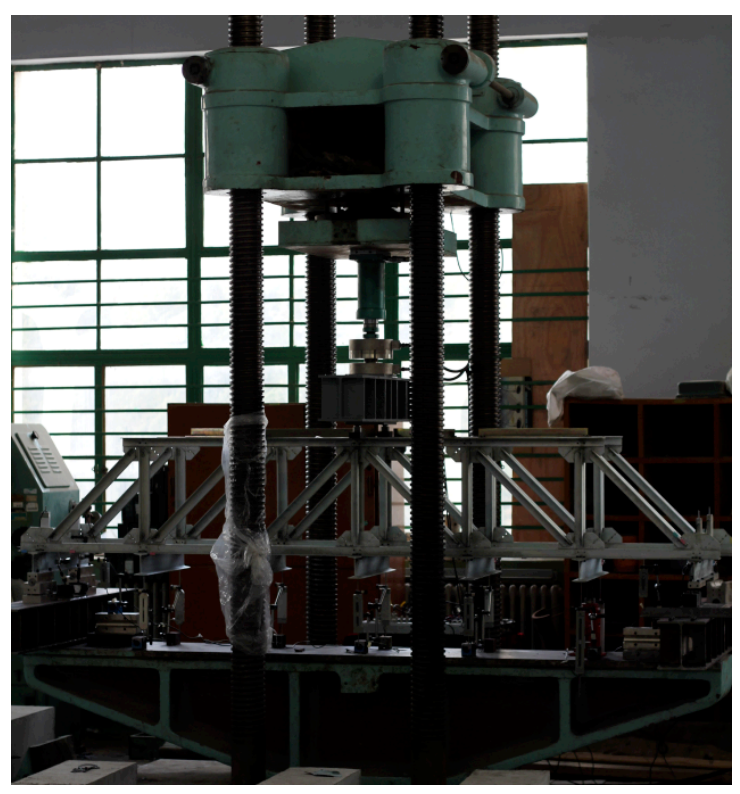

Figure 1 Load test devices of steel truss.

When the test is finished, the random errors of the measured data are rejected according to the method of eliminating the false data and retaining the true data, whose procedure is shown as follow.

(1) The datum of data processing is determined. According to structural mechanical diagram, the deflections on each measuring points are calculated, and so are the average deviations between the theoretical results and the measured data. The results, which are the sum of the theoretical results and the average deviations, are set as the datum of data processing.

(2) The random errors are rejected. Let $\mu$ and $\sigma$ be the mean and standard deviation of the measured data. Because the random errors usually comply with the normal distribution, and the probability that the random errors are larger than $3 \sigma$ is only $0.3 \%$ (Cowan and Dixon (1978)), let $\left[\mu_{u}-3 \sigma_{u}, \mu_{u}+3 \sigma_{u}\right]$ represent the value range of deflections, according to which, the random errors are rejected.

The load-deflection curves of B1 measuring points are drawn on Figure 2, where the solid line and the dash-dot line are the measured data and the processed data. The results show that the 
original records meet linear and stability, and the processed data almost coincides with the reasonable part of the original records, which illustrate that the deflections after data processing are reliable and stable. The other data are consistent with the above data, which proves the static load test data are reliable and stable, and the method of eliminating the false data and retaining the true data is effective. These data will be the basis for model updating.

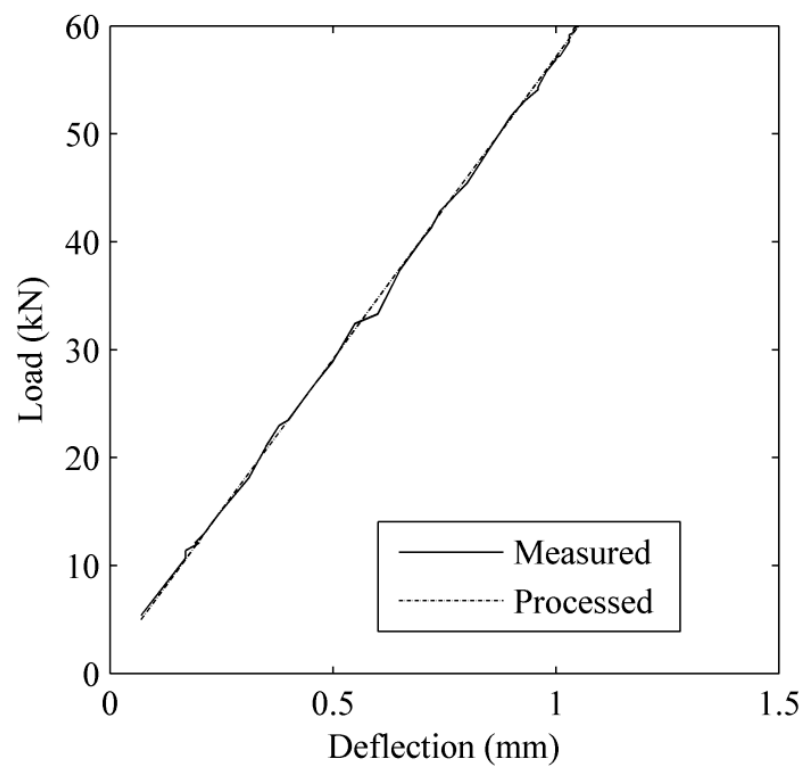

Figure 2 Data processing results of B1 measuring point.

\section{DETERMINATION OF MODEL UPDATING FACTORS}

To observe of the steel truss used for checking the FAMU method, the connections between the gusset plate and its corresponding part on member are slotted and then connected by bolts. When the truss are loaded, effected by the slot, the ability of transferring moment of the members is weakened, which attempts to simulate the similar mechanical property of an ideal truss. Meanwhile, the members are slightly welded with the gusset plates to prevent the failure of the joints.

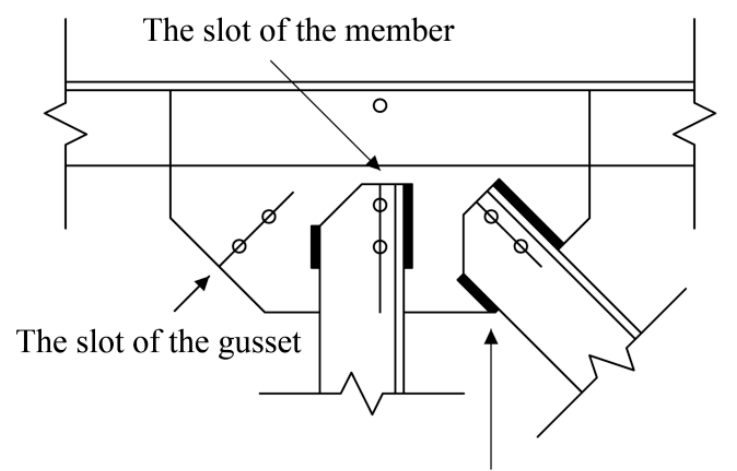

The welding between the member and the gusset

Figure 3 Construction of model joints. 
The detailed construction shown in Figure 3 makes the joints able to transfer axial force and a little moment. Above construction features make the model not able to be simulated by the theoretical frame model or truss model effectively. Therefore, the stiffness of the joints is regarded as the correction factor. Besides, with the truss analyzed, there aren't other factors impacting the model. Therefore, the stiffness of the joints is determined as the correction factors.

\subsection{A variable stiffness beam to simulate model joints}

Aiming at the actual construction of the truss, a variable stiffness beam is proposed to simulate the mechanical property of the model joints, which is shown in Figure 4. For the variable stiffness beam, the two ends represent the model joints, which include the connecting part between the member and the gusset plate. The two ends of the variable stiffness beam are assumed to have the same stiffness as the model joints. The middle part of the variable stiffness beam represents the member, whose stiffness is equal to that of the members. The element stiffness matrix of the variable stiffness beam is derived as follow.

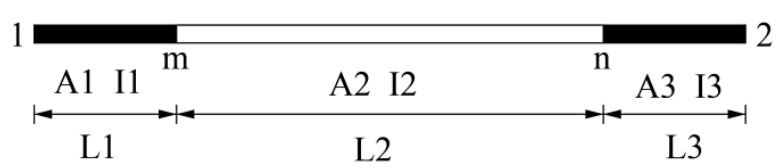

Figure 4 A variable stiffness beam element.

The stiffness matrix of the ith part of the beam element with variable stiffness is

$$
\boldsymbol{k}^{i}=\left(\begin{array}{cccccc}
a & 0 & 0 & -a & 0 & 0 \\
& b & c & 0 & -b & c \\
& & d & 0 & -c & d / 2 \\
& & & a & 0 & 0 \\
& \text { sym. } & & & b & -c \\
& & & & & d
\end{array}\right)^{i}=\left(\begin{array}{cc}
\boldsymbol{k}_{11}^{i} & \boldsymbol{k}_{12}^{i} \\
\boldsymbol{k}_{21}^{i} & \boldsymbol{k}_{22}^{i}
\end{array}\right)
$$

where $a=E A_{i} / l_{i} ; b=12 E I_{i} / l_{i}^{3} ; c=6 E I_{i} / l_{i}^{2} ; d=4 E I_{i} / l_{i},(i=1,2,3)$.

Therefore, the equation of equilibrium of the beam element with variable stiffness is

$$
\left(\begin{array}{cccc}
\boldsymbol{k}_{11}^{1} & \boldsymbol{k}_{12}^{1} & \mathbf{0} & \mathbf{0} \\
\boldsymbol{k}_{21}^{1} & \boldsymbol{k}_{22}^{1}+\boldsymbol{k}_{11}^{2} & \boldsymbol{k}_{12}^{2} & \mathbf{0} \\
\mathbf{0} & \boldsymbol{k}_{21}^{2} & \boldsymbol{k}_{22}^{2}+\boldsymbol{k}_{11}^{3} & \boldsymbol{k}_{12}^{3} \\
\mathbf{0} & \mathbf{0} & \boldsymbol{k}_{21}^{3} & \boldsymbol{k}_{22}^{3}
\end{array}\right)\left(\begin{array}{c}
\boldsymbol{\Delta}_{1} \\
\boldsymbol{A}_{m} \\
\boldsymbol{\Delta}_{n} \\
\boldsymbol{\Delta}_{2}
\end{array}\right)=\left(\begin{array}{c}
\boldsymbol{F}_{1} \\
\mathbf{0} \\
\mathbf{0} \\
\boldsymbol{F}_{2}
\end{array}\right)
$$


where $\boldsymbol{\Delta}_{m}$ and $\boldsymbol{\Delta}_{n}$ are the displacements of $m$ and $n$ nodes in the beam element with variable stiffness; $\boldsymbol{A}_{1}$ and $\boldsymbol{\Delta}_{2}$ represent the displacements of 1 and 2 nodes; $\boldsymbol{F}_{1}$ and $\boldsymbol{F}_{2}$ are node loads.

According to equation (2), the element stiffness matrix of the beam element with variable stiffness is

$$
\left(\begin{array}{cc}
\boldsymbol{k}_{11}^{1}-\boldsymbol{k}_{12}^{1} \overline{\boldsymbol{K}}_{11} \boldsymbol{k}_{21}^{1} & -\boldsymbol{k}_{12}^{1} \overline{\boldsymbol{K}}_{12} \boldsymbol{k}_{12}^{3} \\
-\boldsymbol{k}_{21}^{3} \overline{\boldsymbol{K}}_{21} \boldsymbol{k}_{21}^{1} & \boldsymbol{k}_{22}^{3}-\boldsymbol{k}_{21}^{3} \overline{\boldsymbol{K}}_{22} \boldsymbol{k}_{12}^{3}
\end{array}\right)\left(\begin{array}{c}
\boldsymbol{A}_{1} \\
\boldsymbol{\Delta}_{2}
\end{array}\right)=\left(\begin{array}{c}
\boldsymbol{F}_{1} \\
\boldsymbol{F}_{2}
\end{array}\right),
$$

where $\overline{\boldsymbol{K}}_{i j}(i, j=1,2)$ represents the inverse matrix of the element stiffness matrix of the beam element with variable stiffness; $\boldsymbol{k}_{i j}^{m}(i, j=1,2)$ is the element stiffness matrix of the $m t h(m=1,2,3)$ part of the beam element with variable stiffness.

During the actual computation of the beam element with variable stiffness, let

$$
\alpha=E A_{1} / E A_{2}=E A_{3} / E A_{2} ; \quad \gamma=E I_{1} / E I_{2}=E I_{3} / E I_{2} ; \quad \kappa=L_{1} / L_{2}=L_{3} / L_{2},
$$

where $\alpha, \gamma, \kappa$ are the proportional coefficients of axial stiffness, bending stiffness and joint length between the variable stiffness part and the invariable stiffness part; $E A_{i}, E I_{i}, L_{i}(i=1,2,3)$ are the axial stiffness, the bending stiffness and the joint length of the ith part in Figure 4 , and $\alpha, \gamma, \kappa$ will be the factors of model updating.

\subsection{Determination of the training samples by confidence interval}

For model updating of an actual structure, multi-groups of data are usually measured. Even if there is no effect of noises, there still exists a random deviation among each group of data. In general, only the average of the measured data is used for model updating, but cannot reflect structural whole performances. Therefore, the concept of confidence interval is introduced. Firstly, the confidence interval of the measured data with $95 \%$ confidence probability is calculated. Secondly, the appointed number of training samples for $\mathrm{NN}$ is randomly extracted from the confidence interval of the measured data. Finally, the average of the output of NN is regarded as the result of model updating.

In most cases, the samples comply with normal distribution approximately, so is the measured data assumed. For the samples complying with normal distribution, the confidence interval of their expectation with confidence probability of $1-\alpha$ (Degroot (1989)) is

$$
\left(\bar{X}-\frac{S}{\sqrt{n}} t_{\alpha / 2}(n-1), \bar{X}+\frac{S}{\sqrt{n}} t_{\alpha / 2}(n-1)\right),
$$

where $\bar{X}$ is the statistical average of the samples; $S$ is the standard deviation of the samples; $t_{\alpha / 2}(n-1)$ represents the $t$ distribution; $n$ is sample number.

Latin American Journal of Solids and Structures 11(2014) $019-034$ 


\subsection{Uniform design}

Uniform design is an experimental design method. It carries out the experimental design through a well-designed table. Each table has its own code $U_{n}\left(q^{s}\right)$ or $U_{n}^{*}\left(q^{s}\right)$, in which $U$ represents uniform design; $n$ represents test number; $q$ represents level number; $s$ represents the most factor numbers that the table can arrange. The right-superscript of $U$ with or without * represents two different types of uniform design table. Generally, $U$ with * represents the table has better uniformity and should be used in priority. For example, $U_{29}^{*}\left(29^{6}\right)$ means 29 experiments are going to be done, each factor has 29 levels, the table can arrange experiment with the most 6 factors, it is shown in Table 1. The meaning of $U_{4}^{*}\left(4^{4}\right)$ and $U_{24}^{*}\left(24^{12}\right)$ is similar with $U_{29}^{*}\left(29^{6}\right)$.

Table 1 Uniform design table $U_{29}^{*}\left(29^{6}\right)$.

\begin{tabular}{|c|c|c|c|c|c|c|}
\hline Factor & 1 & 2 & 3 & 4 & 5 & 6 \\
\hline Level 1 & 1 & 13 & 17 & 19 & 23 & 29 \\
\hline Level 2 & 2 & 26 & 3 & 7 & 15 & 27 \\
\hline Level 3 & 3 & 8 & 20 & 26 & 7 & 25 \\
\hline Level 4 & 4 & 21 & 6 & 14 & 30 & 23 \\
\hline Level 5 & 5 & 3 & 23 & 2 & 22 & 21 \\
\hline Level 6 & 6 & 16 & 9 & 21 & 14 & 19 \\
\hline Level 7 & 7 & 29 & 26 & 9 & 6 & 17 \\
\hline Level 8 & 8 & 11 & 12 & 28 & 29 & 15 \\
\hline Level 9 & 9 & 24 & 29 & 16 & 21 & 13 \\
\hline Level 10 & 10 & 6 & 15 & 4 & 13 & 11 \\
\hline Level 11 & 11 & 19 & 1 & 23 & 5 & 9 \\
\hline Level 12 & 12 & 1 & 18 & 11 & 28 & 7 \\
\hline Level 13 & 13 & 14 & 4 & 30 & 20 & 5 \\
\hline Level 14 & 14 & 27 & 21 & 18 & 12 & 3 \\
\hline Level 15 & 15 & 9 & 7 & 6 & 4 & 1 \\
\hline Level 16 & 16 & 22 & 24 & 25 & 27 & 30 \\
\hline Level 17 & 17 & 4 & 10 & 13 & 19 & 28 \\
\hline Level 18 & 18 & 17 & 27 & 1 & 11 & 26 \\
\hline Level 19 & 19 & 30 & 13 & 20 & 3 & 24 \\
\hline Level 20 & 20 & 12 & 30 & 8 & 26 & 22 \\
\hline Level 21 & 21 & 25 & 16 & 27 & 18 & 20 \\
\hline Level 22 & 22 & 7 & 2 & 15 & 10 & 18 \\
\hline Level 23 & 23 & 20 & 19 & 3 & 2 & 16 \\
\hline Level 24 & 24 & 2 & 5 & 22 & 25 & 14 \\
\hline Level 25 & 25 & 15 & 22 & 10 & 17 & 12 \\
\hline Level 26 & 26 & 28 & 8 & 29 & 9 & 10 \\
\hline Level 27 & 27 & 10 & 25 & 17 & 1 & 8 \\
\hline Level 28 & 28 & 23 & 11 & 5 & 24 & 6 \\
\hline Level 29 & 29 & 5 & 28 & 24 & 16 & 4 \\
\hline
\end{tabular}




\section{A STEPWISE UNIFORM DESIGN SCHEME CORRECTS MODEL PARAMETERS}

With the use of the measured deflections of the static load test, the parameters of the truss are corrected by three schemes: a full-factor UD scheme, a nested UD scheme and a stepwise UD scheme.

\subsection{Generation of the stepwise uniform design scheme}

The full-factor UD scheme (FFUD): It makes the variable stiffness coefficients of all members as the correction factors to establish a large-scale UD scheme, and then correct the factors finely. The responses computed by the updated model are coincided with the measured data. However, the problem is that there is a big difference in the corrected factors of the same kind of members, which is not consistent with the actual construction. To analyze the problem, it can be found that each factor, in an UD scheme, is completely independent (Qin et al. (2006)), but the constructions among the same kind of members are similar, which leads to the fact that the FFUD does not include the real solution of model updating. Meanwhile, the solution of an inverse problem is not unique. A pseudo solution, which makes the computed responses in accordance with the real situation but not the real solution of model updating, is gotten by the FFUD.

The nested UD scheme (NUD): Firstly, all members are classified according to their types, and a whole UD scheme with different levels of all members is briefly built by a small-scale UD table. Then, according to the actual constructions, the correction factors on the same level of the same member are imposed some proper constraints so as to make these factors be modified on a small range. By this way, the fine scheme of each level is set up. Finally, the NUD is formed by nesting the fine schemes into the whole UD scheme, which realizes the effect of a large-scale UD scheme by nesting multi small-scale UD schemes into the whole UD scheme, and the constraints will restrict the differences among the same kind of members so that the results of model updating become more reasonable. The results show that the solutions of the NUD are more reasonable than that of the FFUD, but there are still some differences among the same kind of members. To analyze the NUD, it can be found that the NUD only limits the mechanical properties of members on the same level but not restricts that among different levels, which leads to the fact that the solutions of the NUD are not completely reasonable. In order to solve this problem, a stepwise UD scheme is built.

The stepwise UD scheme (SUD): Based on structural mechanical properties, the correction factors are ordered according to their importance, from most to least significant: axial stiffness, bending stiffness and joint length. All members are classified according to their types, and the model is updated by two steps using multi small-scale UD tables.

On the first step, the proportional coefficient of axial stiffness $(\alpha)$ is the principal factor and the other two factors $(\gamma, \kappa)$ are the secondary ones. The input of $\mathrm{NN}$ is deflection, and NN mainly predicts $\alpha$ and incidentally identifies $\gamma, \kappa$. The initial range of each factor is determined as $\alpha \in[0.8,1.2], \gamma \in[0.8,1.2]$ and $\kappa \in[0.18,0.22]$. The UD scheme is arranged by the first, third, and fourth columns of $U_{29}^{*}\left(29^{6}\right)$ listed in Table 1, whose deviation is 0.0914. Then, the test number

Latin American Journal of Solids and Structures 11(2014) $019-034$ 
of the SUD is extended to 116 by changing the position of each factor using $\boldsymbol{U}_{4}^{*}\left(4^{4}\right)$ listed in Table 2. A back-propagation neural network (BPNN) with three layers is built, where the neuron numbers of input layer, hidden layer and output layer are 5, 11 and 3 respectively, and the steepest descent method with momentums is applied to the training of BPNN. The error and maximum frequency of training are 0.001 and 10000 respectively. The mean squared error in the process of training is shown in Figure 5. Finally, the confidence interval of the measured data is computed according to Equation (5). Forty-five groups of samples are randomly extracted from the confidence interval and input into the trained BPNN. Forty-five groups of model parameters are predicted, whose averages are taken as the results of model updating on the first step, which are $\alpha=0.9074$, $\gamma=0.8830, \kappa=0.1941$.

Table 2 Uniform design table $\boldsymbol{U}_{4}^{*}\left(4^{4}\right)$.

\begin{tabular}{ccccc}
\hline Factor & 1 & 2 & 3 & 4 \\
\hline Level 1 & 1 & 2 & 3 & 4 \\
Level 2 & 2 & 4 & 1 & 3 \\
Level 3 & 3 & 1 & 4 & 2 \\
Level 4 & 4 & 3 & 2 & 1 \\
\hline
\end{tabular}

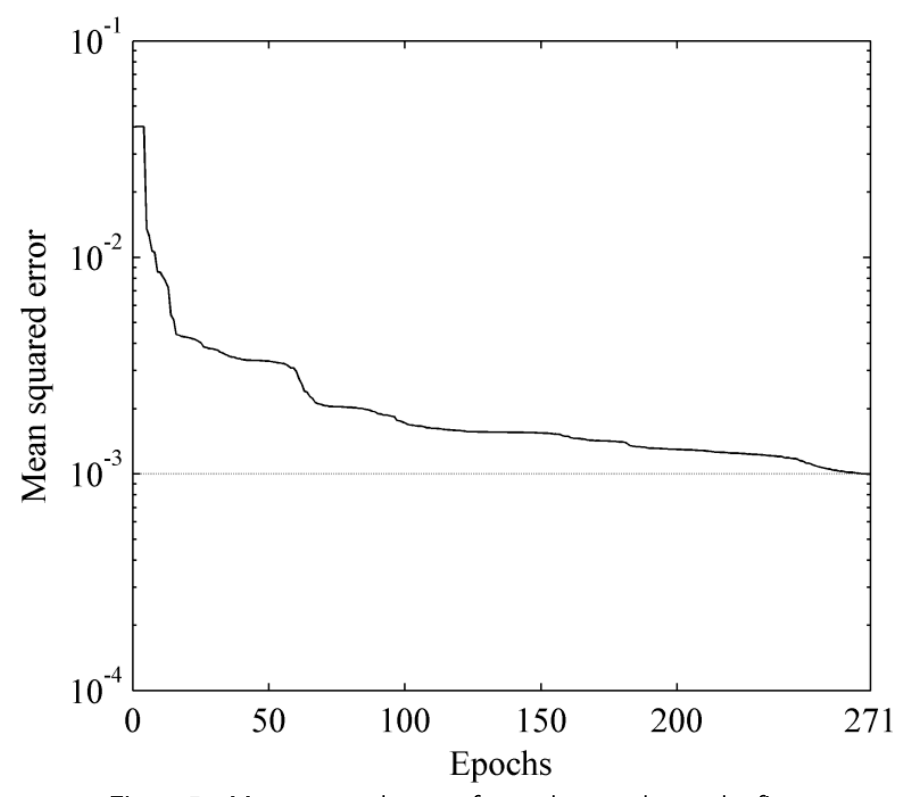

Figure 5 Mean squared error of neural networks on the first step.

On the second step, the proportional coefficients of each kind of members are reasonably adjusted according to the results of the first step, which means the correlations among factors are considered. The factors of model updating are changed according to the ratio of the results between the first and the second steps, which are denoted by $c_{1} \sim c_{12}$. For example, 


$$
c_{1}=\frac{\alpha_{s}}{\alpha_{1}} \quad c_{2}=\frac{\gamma_{s}}{\gamma_{1}} \quad c_{3}=\frac{\kappa_{s}}{\kappa_{1}}
$$

where $\alpha_{1}, \gamma_{1}, \kappa_{1}$ are the results of model updating on the first step; $s$ represents upper chord; $c_{1} \sim c_{3}$ are the ratio of the results of model updating between the first and the second steps.

According to structural actual constructions, the initial value ranges of factors are $c_{1} \in[1.0,1.2]$, $c_{2} \in[1.0,1.2], c_{3} \in[0.9,1.1]$, and the meaning and value of the other nine factors $c_{4} \sim c_{12}$ are similar to $c_{1} \sim c_{3}$. At the same time, the UD scheme is built by $U_{24}^{*}\left(24^{12}\right)$ listed in Table 3 .

Table 3 Uniform design table $U_{24}^{*}\left(24^{12}\right)$.

\begin{tabular}{|c|c|c|c|c|c|c|c|c|c|c|c|c|}
\hline Factor & 1 & 2 & 3 & 4 & 5 & 6 & 7 & 8 & 9 & 10 & 11 & 12 \\
\hline Level 1 & 1 & 6 & 7 & 8 & 9 & 11 & 12 & 13 & 16 & 17 & 18 & 24 \\
\hline Level 2 & 2 & 12 & 14 & 16 & 18 & 22 & 24 & 1 & 7 & 9 & 11 & 23 \\
\hline Level 3 & 3 & 18 & 21 & 24 & 2 & 8 & 11 & 14 & 23 & 1 & 4 & 22 \\
\hline Level 4 & 4 & 24 & 3 & 7 & 11 & 19 & 23 & 2 & 14 & 18 & 22 & 21 \\
\hline Level 5 & 5 & 5 & 10 & 15 & 20 & 5 & 10 & 15 & 5 & 10 & 15 & 20 \\
\hline Level 6 & 6 & 11 & 17 & 23 & 4 & 16 & 22 & 3 & 21 & 2 & 8 & 19 \\
\hline Level 7 & 7 & 17 & 24 & 6 & 13 & 2 & 9 & 16 & 12 & 19 & 1 & 18 \\
\hline Level 8 & 8 & 23 & 6 & 14 & 22 & 13 & 21 & 4 & 3 & 11 & 19 & 17 \\
\hline Level 9 & 9 & 4 & 13 & 22 & 6 & 24 & 8 & 17 & 19 & 3 & 12 & 16 \\
\hline Level 10 & 10 & 10 & 20 & 5 & 15 & 10 & 20 & 5 & 10 & 20 & 5 & 15 \\
\hline Level 11 & 11 & 16 & 2 & 13 & 24 & 21 & 7 & 18 & 1 & 12 & 23 & 14 \\
\hline Level 12 & 12 & 22 & 9 & 21 & 8 & 7 & 19 & 6 & 17 & 4 & 16 & 13 \\
\hline Level 13 & 13 & 3 & 16 & 4 & 17 & 18 & 6 & 19 & 8 & 21 & 9 & 12 \\
\hline Level 14 & 14 & 9 & 23 & 12 & 1 & 4 & 18 & 7 & 24 & 13 & 2 & 11 \\
\hline Level 15 & 15 & 15 & 5 & 20 & 10 & 15 & 5 & 20 & 15 & 5 & 20 & 10 \\
\hline Level 16 & 16 & 21 & 12 & 3 & 19 & 1 & 17 & 8 & 6 & 22 & 13 & 9 \\
\hline Level 17 & 17 & 2 & 19 & 11 & 3 & 12 & 4 & 21 & 22 & 14 & 6 & 8 \\
\hline Level 18 & 18 & 8 & 1 & 19 & 12 & 23 & 16 & 9 & 13 & 6 & 24 & 7 \\
\hline Level 19 & 19 & 14 & 8 & 2 & 21 & 9 & 3 & 22 & 4 & 23 & 17 & 6 \\
\hline Level 20 & 20 & 20 & 15 & 10 & 5 & 20 & 15 & 10 & 20 & 15 & 10 & 5 \\
\hline Level 21 & 21 & 1 & 22 & 18 & 14 & 6 & 2 & 23 & 11 & 7 & 3 & 4 \\
\hline Level 22 & 22 & 7 & 4 & 1 & 23 & 17 & 14 & 11 & 2 & 24 & 21 & 3 \\
\hline Level 23 & 23 & 13 & 11 & 9 & 7 & 3 & 1 & 24 & 18 & 16 & 14 & 2 \\
\hline Level 24 & 24 & 19 & 18 & 17 & 16 & 14 & 13 & 12 & 9 & 8 & 7 & 1 \\
\hline
\end{tabular}

The factors of model updating are updated in the same way as on the first step. The mean squared error of BPNN on the second step is shown in Figure 6. The results of model updating on the second step are listed in Table 4.

Latin American Journal of Solids and Structures 11(2014) 019 - 034 
Table 4 Results of model updating on the second step.

\begin{tabular}{cccccccccccc}
\hline$c_{1}$ & $c_{2}$ & $c_{3}$ & $c_{4}$ & $c_{5}$ & $c_{6}$ & $c_{7}$ & $c_{8}$ & $c_{9}$ & $c_{10}$ & $c_{11}$ & $c_{12}$ \\
\hline 1.127 & 1.179 & 1.077 & 1.119 & 1.169 & 1.087 & 0.717 & 0.592 & 1.278 & 0.699 & 0.574 & 1.293 \\
\hline
\end{tabular}

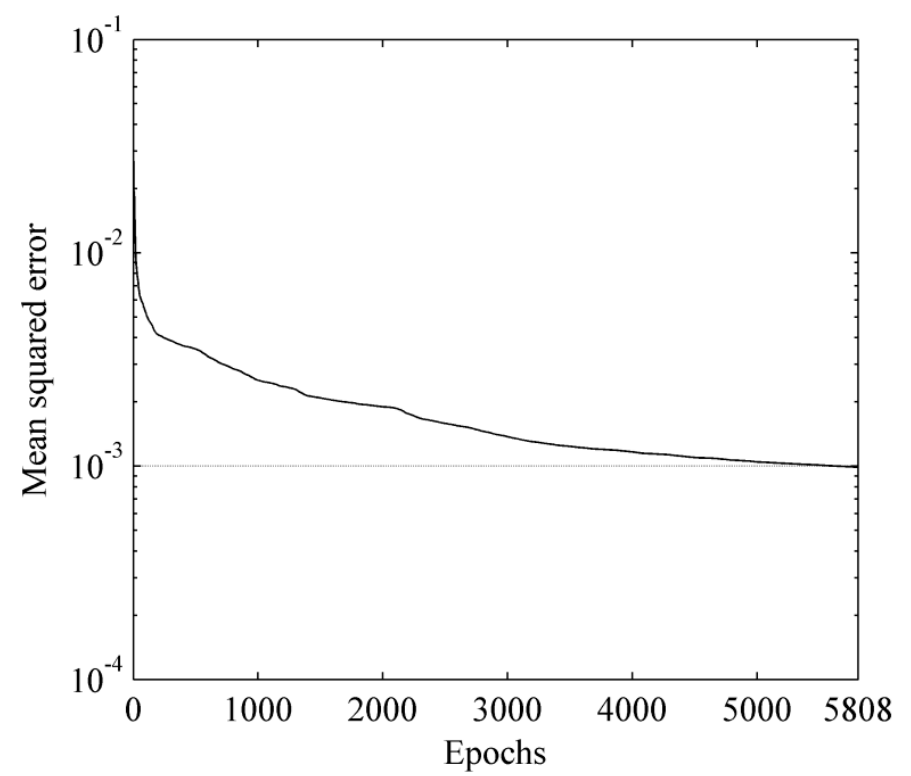

Figure 6 Mean squared error of neural networks on the second step.

\subsection{Results of model updating}

The final results of the SUD are listed in Table 5, where s, sg, xg and x represent upper chord, vertical member, diagonal member and bottom chord respectively. From this table it can be noted that the proportional coefficients of axial stiffness and bending stiffness of chords are all greater than 1 , which illustrates that the stiffness of joints of chords is greater than that of members; the proportional coefficients of axial stiffness and bending stiffness of web members are all less than 1, which agrees with the actual situation; the proportional coefficients of joint length of all members belong to $0.2 \sim 0.25$, which are close to the size of the gussets.

Table 5 Results of the stepwise uniform design scheme.

\begin{tabular}{cccccccccccc}
\hline$\alpha_{\mathrm{s}}$ & $\gamma_{\mathrm{s}}$ & $x_{\mathrm{s}}$ & $\alpha_{\mathrm{sg}}$ & $\gamma_{\mathrm{sg}}$ & $x_{\mathrm{sg}}$ & $\alpha_{\mathrm{xg}}$ & $\gamma_{\mathrm{xg}}$ & $x_{\mathrm{xg}}$ & $\alpha_{\mathrm{x}}$ & $\gamma_{\mathrm{x}}$ & $\chi_{\mathrm{x}}$ \\
\hline 1.023 & 1.041 & 0.209 & 0.651 & 0.523 & 0.248 & 0.634 & 0.507 & 0.251 & 1.015 & 1.032 & 0.211 \\
\hline
\end{tabular}

In order to check the accuracy of model updating, the deflections of each measuring points by the updated model are computed in the condition that a $60 \mathrm{kN}$ concentrated force is imposed on the mid-span of the upper chords. The comparison between the computed and measured deflections is shown in Figure 7, where the dot line, the dash-dot line and the solid line are the deflections computed by the non-updated model, computed by the updated model and the measured ones respec- 
tively. From Figure 7, it can be found that the computed results of the updated model are better than that of the non-updated model obviously and closer to the measured deflections.

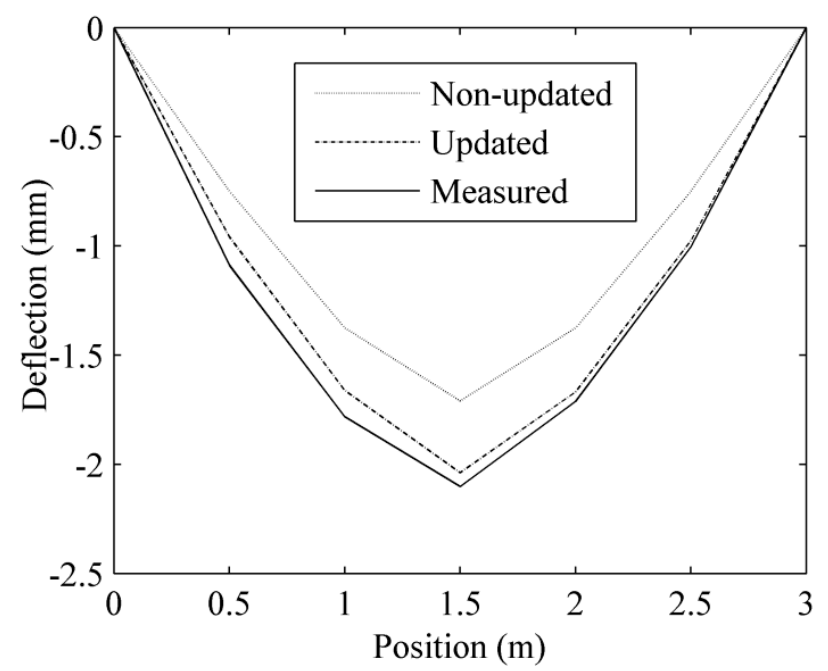

Figure 7 Comparison of deflections before and after model updating on the mid-span loading.

Further, the deflections under two-point symmetrical loading are measured and listed in Table 6 . Figure 8 illustrates the condition of two-point symmetrical loading. And the deflections before and after model updating with $\mathrm{F}=30 \mathrm{kN}$ are compared, the result is shown in Figure 9 . The result shows that the updated deflections are in accordance with the measured ones after changing the loading condition, namely, the results of model updating in this paper are reliable.

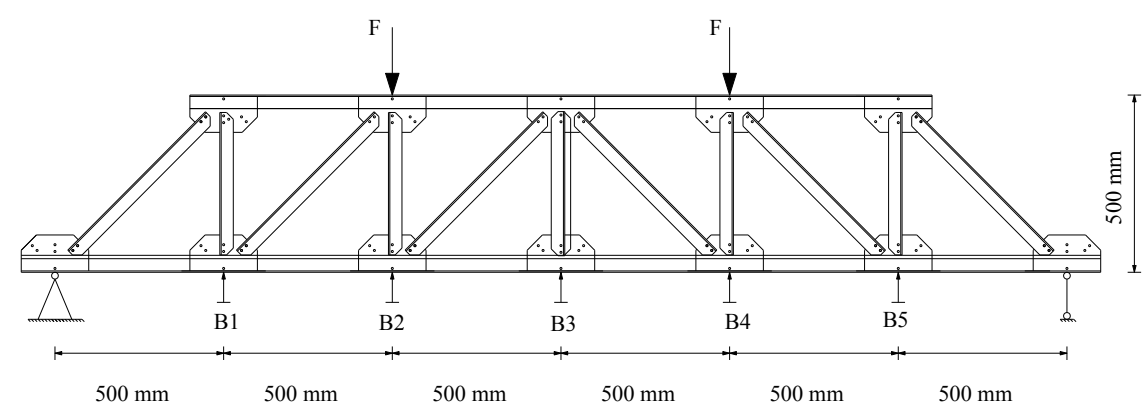

Figure 8 Two-point symmetrical loading. 
Table 6 Deflections of two-point symmetrical loading (Unit: $\mathrm{mm}$ ).

\begin{tabular}{|c|c|c|c|c|c|}
\hline $\mathrm{F}(\mathrm{kN})$ & B1 & $\mathrm{B} 2$ & B3 & $\mathrm{B} 4$ & B5 \\
\hline 2.5 & 0.026 & 0.029 & 0.024 & 0.044 & 0.003 \\
\hline 5.0 & 0.105 & 0.154 & 0.160 & 0.170 & 0.073 \\
\hline 7.5 & 0.184 & 0.280 & 0.296 & 0.295 & 0.144 \\
\hline 10.0 & 0.264 & 0.405 & 0.432 & 0.420 & 0.214 \\
\hline 12.5 & 0.343 & 0.531 & 0.569 & 0.545 & 0.284 \\
\hline 15.0 & 0.422 & 0.656 & 0.705 & 0.670 & 0.354 \\
\hline 17.5 & 0.501 & 0.782 & 0.841 & 0.795 & 0.425 \\
\hline 20.0 & 0.580 & 0.907 & 0.977 & 0.921 & 0.495 \\
\hline 22.5 & 0.659 & 1.033 & 1.114 & 1.046 & 0.565 \\
\hline 25.0 & 0.738 & 1.158 & 1.250 & 1.171 & 0.635 \\
\hline 27.5 & 0.817 & 1.284 & 1.386 & 1.296 & 0.706 \\
\hline 30.0 & 0.896 & 1.409 & 1.522 & 1.421 & 0.776 \\
\hline
\end{tabular}

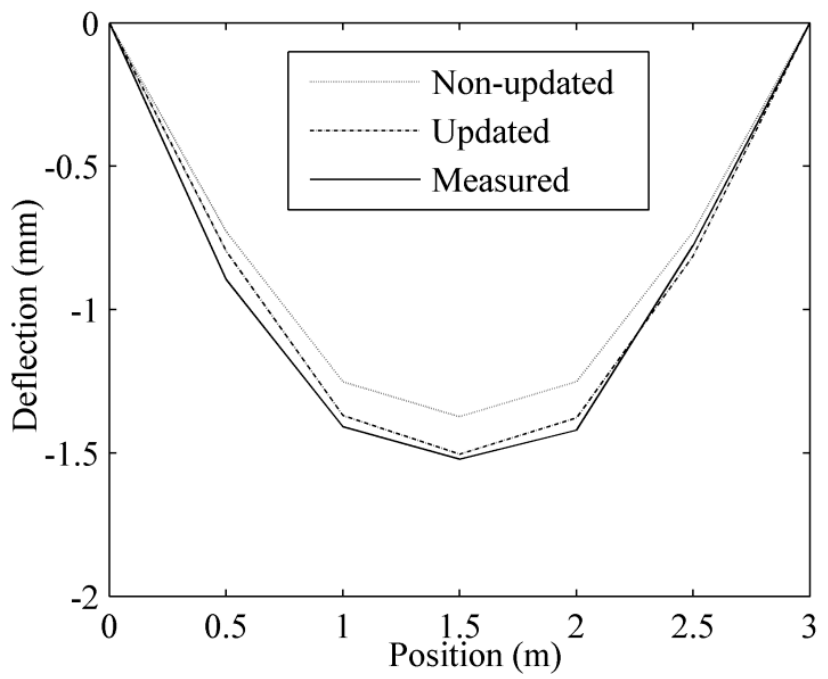

Figure 9 Comparison of deflections before and after model updating on two-point symmetrical loading.

\subsection{Forward analytical model updating extends to complex structures}

The projects that are needed to be corrected are usually large-scale complex structures. Above studied object is a small-scale truss, which model updating has a difference with that of the largescale complex structures. The most difference of model updating between small-scale structures and large-scale ones lies in that large-scale structures have more factors needed to be corrected and the types of factors are more comprehensive. When there are many factors needed to be updated, the first difficulty is how to establish large-scale uniform design schemes. The scale of factors by the available uniform design tables is less than 50. The authors have studied how to build large-scale uniform design tables and their application tables, and use the centered $L_{2}$ deviation to assess their uniformity. The FFUD is built based on a large-scale uniform design table. But the large-scale uni- 
form design scheme may not be able to obtain a satisfied result, which reason lies in that each factor of UD is completely independence and uniformly dispersed. However, there are constraints among factors for actual structures, which are proven by the results of the FFUD.

For the model updating of large-scale complex structures, not only the factors are increased, but also the types of factors are extended. Model updating actually includes two categories of factors: structural design parameters and the elements of stiffness, damping and mass matrix. The reason why the mechanical models of complex structures are not accurate may lies in the inaccurate design parameters or the incorrect elements of the matrix, which are induced by mechanical hypothesis, simplified modeling and ill-defined boundary condition. In most cases, it is difficult to realize model updating that only correct design parameters or the elements of the matrix. A feasible approach to solve the problem of model updating of large-scale complex structures is that integrates principal factor analysis, stepwise uniform design and optimum seeking method (Hua and Halberstam (1983)) into the FAMU method based on UD.

Let the design parameters of large-scale complex structures be $\boldsymbol{p}$ and the stiffness matrix be $\boldsymbol{K}$. $\boldsymbol{\omega}$ and $\boldsymbol{u}$ represent the computed frequency and deformation of structural finite element model; $\overline{\boldsymbol{\omega}}$ and $\overline{\boldsymbol{u}}$ are structural measured frequency and actual deformation.

$$
\begin{aligned}
& \boldsymbol{p}=\boldsymbol{p}_{0}+\sum_{i=1}^{n} a_{i} \boldsymbol{p}_{\mathrm{a}_{i}}+\sum_{j=1}^{m} b_{j} \boldsymbol{p}_{\mathrm{b}_{j}}, \\
& \boldsymbol{K}=\boldsymbol{K}_{0}+\sum_{t=1}^{o} c_{t} \boldsymbol{K}_{\mathrm{c}_{t}}+\sum_{r=1}^{q} d_{r} \boldsymbol{K}_{\mathrm{d}_{r}},
\end{aligned}
$$

where $a_{i}, b_{j}, c_{t}$ and $d_{r}$ are the factors needed to be corrected; $\boldsymbol{p}_{0}$ and $\boldsymbol{K}_{0}$ are the initial value of structural design parameters and stiffness matrix; $\boldsymbol{p}_{\mathrm{a}_{i}}$ and $\boldsymbol{K}_{\mathrm{c}_{t}}$ are the sensitivity matrix of the design parameters and stiffness matrix; $\boldsymbol{p}_{\mathrm{b}_{j}}$ and $\boldsymbol{K}_{\mathrm{d}_{r}}$ represent the difference of the model updating results from the forward step to the last one.

1) The significance of $a_{i}, b_{j}, c_{t}$ and $d_{r}$ for model updating is determined by the perturbation method, which is actually to determine the principal factor.

$$
\begin{aligned}
& \frac{\partial \omega}{\partial a_{i}}=\frac{\omega_{1}-\omega_{0}}{\Delta a_{i}}, \frac{\partial \omega}{\partial b_{j}}=\frac{\omega_{1}-\omega_{0}}{\Delta b_{j}} \\
& \frac{\partial \boldsymbol{u}}{\partial c_{t}}=\frac{\boldsymbol{u}_{1}-\boldsymbol{u}_{0}}{\Delta c_{t}}, \frac{\partial \boldsymbol{u}}{\partial d_{r}}=\frac{\boldsymbol{u}_{1}-\boldsymbol{u}_{0}}{\Delta d_{r}}
\end{aligned}
$$

The actual computational method is to make the factors take place a unit perturbation according to formula (8), so that we can get the rate of change of frequency and deformation, while the factor with the maximum rate of change is the principal factor.

2) Multi small-scale UD schemes are built according to the significance of the factors. When determining the initial value range of each factor, structural actual construction should be considered

Latin American Journal of Solids and Structures 11(2014) $019-034$ 
to impose some appropriate constraints. Meanwhile, the initial value range should be large enough to cover the real solution of model updating, and then each factor is updated stepwise.

3) On each step, a certain number of samples are random selected from the confidence interval of the measured frequency and deformation, and input into the trained $\mathrm{NN}$ to identify multi-group of factors. The averages of the identified factors are as the results of model updating on this step.

4) When all factors have been corrected, the error of model updating will be computed to determine whether meets the requirement of model updating or not. If it cannot meet the requirement, structural model will be corrected until meet the requirement.

5) When there are a great many factors for a large-scale complex structure, we should employ the optimal seeking method to fix the secondary factors and only update the most important factor according to the steps from 1) to 4). After the first principal factor is successfully corrected, the second principal factor will be updated. And we should correct each factor one by one and finally realize to update the finite element model of the large-scale complex structure.

\section{CONCLUSIONS}

This paper updates a steel truss model and summarizes a model updating method using stepwise uniform design schemes considered primary factors. The result shows that the primary factors of model updating are determined correctly; the stepwise updating strategy based on primary factor analysis can use uniform design in small scale to update the model parameters of the steel truss. According to the calculation result, the following conclusion can be reached.

1. For model updating, the most important aspect is to determine the model updating factor correctly. Namely, according to the difference between the responses of the non-updated model and those of the actual structure, we should establish an improved model which must contain the primary factors of model updating and can reflect structural mechanical characters correctly.

2. The value ranges of factors which will be updated should be large enough to cover the actual solution of model updating. Otherwise, the neural networks which are trained by uniform design schemes cannot predict and identify the parameters of the model correctly.

3. In order to reduce the workload and update structural models, primary and secondary factors should be identified clearly. And the stepwise updating strategy with small-scale uniform design schemes should be adopted to update the model parameters.

4. After determining the model updating factor and level reasonably, the model updating method which is proposed in this paper can overcome the influences of uncertain and incomplete measured information and is able to update the model parameters.

Acknowledgements Support from the Nature Science Foundation of China (NSFC), under grant numbers 50978078 and 51208150, is greatly acknowledged. 


\section{References}

Ataei, S., Aghakouchak, A. A., Marefat, M. S., Mohammadzadeh, S., (2005). Sensor fusion of a railway bridge load test using neural networks. Expert Systems with Application 29: 678-683.

Atalla, M. J. and Inman, D. J., (1998). On model updating using neural networks. Mechanical Systems and Signal Processing 12: 135-161.

Bakir, P. G., Reynders, E., Roeck, G. D., (2008). An improved finite element model updating method by the global optimization technique 'Coupled Local Minimizers'. Computers and Structures 86: 1339-1352.

Basağa, H. B., Türker, T., Bayraktar, A., (2011). A model updating approach based on design points for unknown structural parameters. Applied Mathematical Modelling 35: 5872-5883.

Chang, C. C., Chang, T. Y. P., Xu, Y. G., To, W. M., (2002). Selection of training samples for model updating using neural networks. Journal of Sound and Vibration 249: 867-883.

Cheng, J., (2010). An artificial neural network based genetic algorithm for estimating the reliability of long span suspension bridges. Finite Elements in Analysis and Design 46: 658-667.

Cowan, H. J. and Dixon, J., (1978). Building science laboratory manual, Applied Science Publishers (Virginia).

Davoodi, M. R., Amiri, J. V., Gholampour, S., Mostafavian, S. A., (2012). Determination of nonlinear behavior of a ball joint system by model updating. Journal of Constructional Steel Research 71: 52-62.

Degroot, M. H., (1989). Probability and statistics (2nd edition), Addison-Wesley Publishing Company (Massachusetts).

Guajardo, J. A., Weber, R., Miranda, J., (2010). A model updating strategy for predicting time series with seasonal patterns. Applied Soft Computing 10: 276-283.

Hua, L. G. and Halberstam, H., (1983). Loo-keng Hua selected papers, Springer Verlag (New York).

Khanmirza, E., Khaji, N., Majd, V. J., (2011). Model updating of multistory shear buildings for simultaneous identification of mass, stiffness and damping matrices using two different soft-computing methods. Expert Systems with Application 38: 5320-5329.

Lu, Y. and Tu, Z. G., (2004). A two-level neural network approach for dynamic FE model updating including damping. Journal of Sound and Vibration 275: 931-952.

Qin, H., Zhang, S. L., Fang, K. T., (2006). Constructing uniform designs with two- or three- level*. Acta Mathematica Scientia 26: 451-459.

Saaty, T. L. and Vargas, L. G., (1979). Estimating technological coefficients by the analytic hierarchy process. Socio-Economic Planning Sciences 13: 333-336.

Unger, J. F. and Könke, C., (2011). An inverse parameter identification procedure assessing the quality of the estimates using Bayesian neural networks. Applied Soft Computing 11: 3357-3367.

Zadeh, L. A., (1965). Fuzzy sets. Information and Control 8: 338-353.

Zhang, S. L., Chen, S. F., Wang, H. D., Wang, W., (2011). Evaluating identification indices on damage detection by a fuzzy analytical hierarchy process. 2011 International Conference on Structures and Building Materials, Guangzhou. TTP: 2934-2940.

Latin American Journal of Solids and Structures 11(2014) $019-034$ 